\title{
A NOTE ON HYPONORMAL OPERATORS
}

\section{ROBERT WHITLEY}

ABSTRACT. A short, elementary proof that a hyponormal operator with a compact imaginary part is normal.

We give a short elementary proof that a hyponormal operator $T$ $\left(T^{*} T \geq T T^{*}\right)$ which has a compact imaginary part is normal. This is known; for example it follows directly from Putnam's deep results [3, Theorem 3.2.1, p. 42]. As the referee kindly points out, it is also possible to reduce to the case of real spectrum whence the result follows from the fact that the numerical range equals the convex hull of the spectrum. The corollary that a compact hyponormal operator must be normal was demonstrated by Ando [1], Putnam [4], and Stampfli [5].

Theorem. A byponormal operator, on a Hilbert space, which bas a compact imaginary part is normal.

Proof. Let $T$ be the operator, and let $M=\left\{x:\|T x\|=\left\|T^{*} x\right\|\right\}$. As Stampfli notes [6, Lemma 3, p. 474], since $T$ is hyponormal the vector $x$ is in $M$ iff

$$
T T^{*} x=T^{*} T x
$$

To see this we only need observe, as in [6], that for $x$ in $M$ and any $y$,

$$
\left|\left(\left(T^{*} T-T T^{*}\right) x \mid y\right)\right|^{2} \leq\left(\left(T^{*} T-T T^{*}\right) y \mid y\right)\left(\left(T^{*} T-T T^{*}\right) x \mid x\right)=0,
$$

by the Schwarz inequality for positive operators; as $y$ is arbitrary (1) follows. Thus $M$ is a closed subspace.

Let $T=A+i B$, where $A$ is the selfadjoint real part of $T$ and $B$ is the selfadjoint compact imaginary part of $T$. For each eigenvalue $\lambda$ of $B$ and a corresponding eigenvector $x$, we have, as $A+i \lambda$ is normal and $\lambda$ is real, 


$$
\|T x\|=\|(A+i \lambda) x\|=\|(A+i \lambda) * x\|=\|(A-i \lambda) x\|=\|(A-i B) x\|=\left\|T^{*} x\right\| .
$$

Thus the subspace $M$ contains each eigenspace of $B$. These eigenspaces are total, and so $M$ must be the entire space and the operator $T$ must be normal.

\section{REFERENCES}

1. T. Ando, On hyponormal operators, Proc. Amer. Math. Soc. 14 (1963), 290-291. MR 26\#2884.

2. S. Berberian, A note on hyponormal operators, Pacific J. Math. 12 (1962), 1171-1175. MR $26 \# 6771$.

3. C. Putnam, Commutation properties of Hilbert space operators and related topics, Ergebnisse der Mathematik und ihrer Grenzgebiete, Band 36, Springer-Verlag, New York, 1967. MR $36 \# 707$.

4. - On the structure of semi-normal operators, Bull. Amer. Math. Soc. 69 (1963), 818-819. MR 29 \#3886.

5. J. Stampfli, Hyponormal operators, Pacific J. Math. 12 (1962), 1453-1458. MR $26 \# 6772$.

6. - Hyponormal operators and spectral density, Trans. Amer. Math. Soc. 117 (1965), 469-476. MR. 30 \#3375.

DEP ARTMENT OF MATHEMATICS, UNIVERSITY OF CALIFORNIA AT IRVINE, IRVINE, CALIFORNIA 92664 